\title{
The National Interventional Council meeting 2021
}

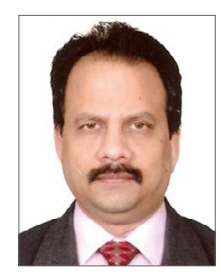

Janakiraman Ezhilan*, MD, DM, FNB; Vasu Nandhakumar, MD, DNB, FNB

Institute of Cardio-Vascular Diseases, The Madras Medical Mission Hospital, Chennai, Tamil Nadu, India

The interventional wing of the Cardiological Society of India was established in 1985, eight years after the first angioplasty procedure on a coronary artery was performed by the German-born physician, Andreas Gruentzig in 1977. Though news of the feasibility of treating coronary artery disease interventionally spread across the globe like wildfire soon after the first procedure, it took some time to construct a dedicated interventional subspeciality of cardiology in India. However, active learning of that new technique and future visions of interventional cardiology were "up and running" inside one of the most prestigious and oldest organisations in India, the Cardiological Society of India (CSI). The CSI was founded 73 years ago in April 1948, soon after independence, a year earlier than the American College of Cardiology. The first meeting of cardiologists who had an interest in interventions assembled for an academic feast in 1985 at Bombay (currently known as Mumbai). The doyens of Indian cardiology named that academic feast the "PTCA Registry Meet". Unfortunately, in the month of October that same year, the airplane that Dr Gruentzig was piloting in stormy weather crashed. The revolution in the treatment of coronary artery disease created by Dr Gruentzig was carefully handed over to successive generations with improvement year after year through the PTCA Registry Meet in India, which was renamed in 1999 the "Midterm National Interventional Cardiology Conference". The current name - the "National Interventional Council" (NIC) - was adopted by the leading interventional cardiologists of India. Traditionally, the NIC organises a midterm meeting in the month of April and an interventional workshop, along with the annual CSI meeting, during
December. It is currently estimated that an average of 1,500 interventional cardiologists are working in 4,185 cardiac catheterisation laboratories (CCL) in India. Appreciating the growing needs of this subspeciality, the NIC has decided to expand itself by creating its own wings, namely coronary, peripheral, structural and paediatric interventions. The NIC is interested in long-term partnerships with regional and international societies. Currently, the NIC, via the CSI, is holding dedicated sessions in the following international meetings, namely CCT Japan, EuroPCR, TCT, TCTAP. The NIC meetings not only attract the enthusiastic brains of India, but also reach out to other south Asian countries such as Nepal, Bangladesh and Sri Lanka. Currently, its membership is close to 4,000 .

The NIC functions under the governance of an organising committee which is selected annually by the CSI members. The CSINIC meeting is rotated among the state CSI branches every year.

The NIC collects data from CCL across India and these data are analysed every year and presented at the annual meeting. Online proformas are available, through which the procedural data from various centres are uploaded to a server. In order to avoid missing data, the NIC also collects data from the National Pharmaceutical Pricing Authority (NPPA), CCL, manufacturers and medical device companies. Duplicate data from multiple sources are eliminated before analysis. The state-wise contribution of interventional cardiac procedures in India is shown in Figure 1. The NIC aims to capture real-time data; the initial steps to execute this have been started. Currently, the data submitted online represent approximately $70 \%$ of CCL in India. Next year's target should be to cover 


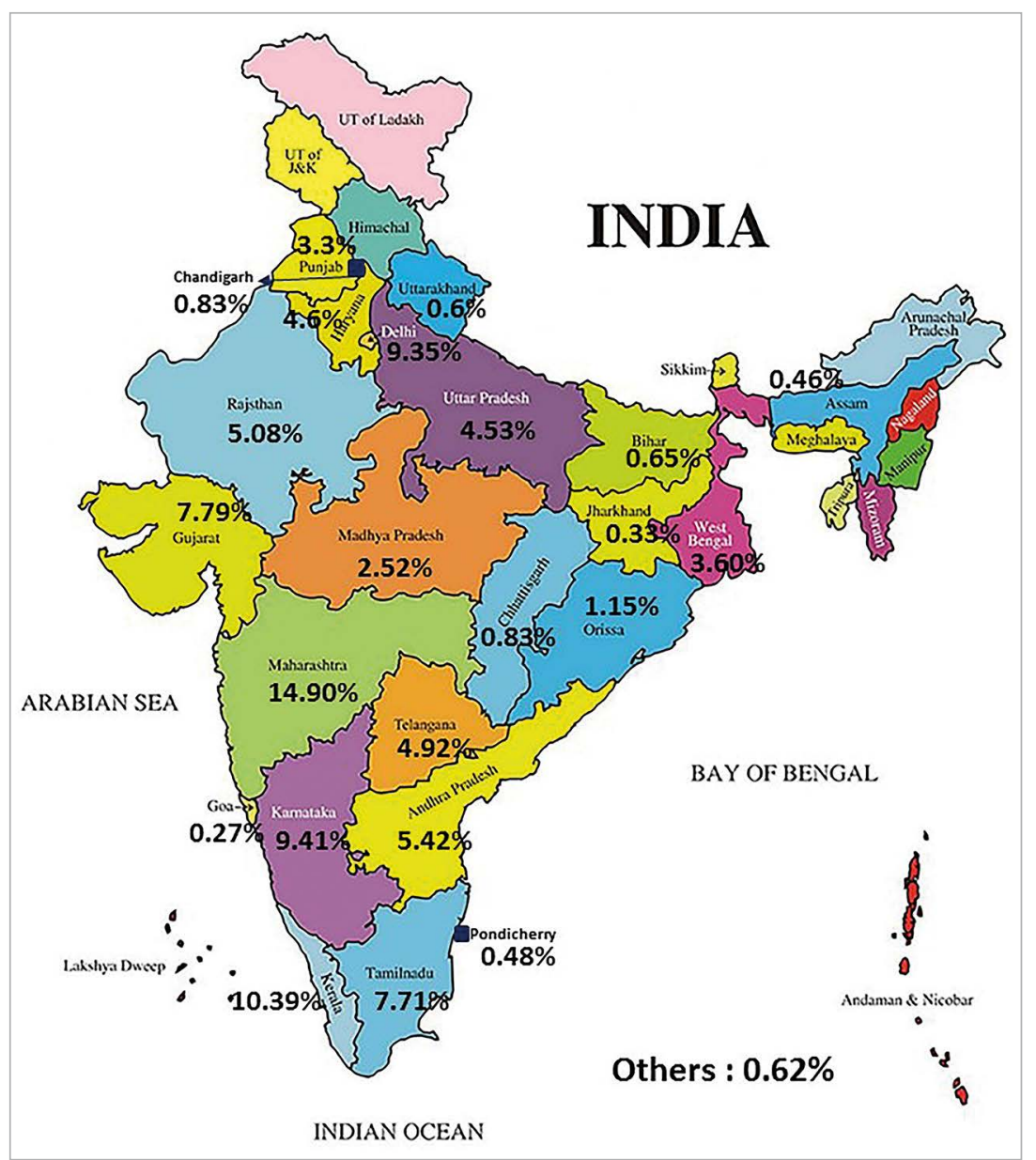

Figure 1. The state-wise contribution of interventional cardiac procedures in India. UT: union territory

an additional $10 \%$ of the CCL of the country. With the expanding number of transcatheter valve interventions, namely transcatheter aortic valve implantations, MitraClip procedures, transcatheter tricuspid valve interventions, and transcatheter valve-in-valve implantations, the NIC plans to start respective registries.

NIC 2021 will take place on $24^{\text {th }}$ and $25^{\text {th }}$ July as a virtual meeting at the end of the second wave of the COVID-19 pandemic. The programme will take place over two days for a total of 24 hours from $4 \mathrm{pm}$ to $10 \mathrm{pm}$ Indian standard time in two theatres (12 hours per day). Two theatres with tightly packed programmes are planned, one dedicated to coronary interventions and the other to structural interventions. The ultimate challenge of this NIC 2021 meeting will be to keep the real flavour of the original three-day meeting with four theatres per day but in a timeconstrained programme of 24 hours. The scientific programme will throw light on cutting-edge technologies such as transcatheter valve implantation and repair, robotic interventions, coronary lithotripsy, excimer laser atherectomy, orbital atherectomy, endovascular aortic interventions, intravascular imaging and coronary physiology. The flagship programme of the NIC is the fellow's course, where the primary focus will be on budding future Gruentzigs. The basic concepts of interventional cardiology will be discussed by national and international prodigies. There is also a live-in-box session, a scenario that not only closely mimics live case transmission, but also stimulates neurotransmitter secretions at the synaptic junctions of audiences. Every session will be followed by a brief discussion of the relevant topic by panellists and chairpersons. The unanswered questions in the chat box will be collected and the answers will be posted on the screen within an hour after completion of the respective session.

The mission of the NIC with its association with AsiaIntervention is to promote research and publications in the field of interventional cardiology. In particular, the NIC makes every effort to create opportunities for domestic researchers to interact with the leading international legends. The NIC works as a bridge to bring the latest technologies to India in real time in the field of interventional cardiology, coming from various parts of the world. The NIC also plays an important role in the country's health policy making. We welcome all faculties and delegates to NIC 2021 and we assure you of a brainstorming academic feast.

\section{Conflict of interest statement}

J. Ezhilan is the organising secretary of NIC 2021. The other author has no conflicts of interest to declare. 